\title{
USOS DE LA EVALUACION DE LOS APRENDIZAJES POR DOCENTES DE ESCUELAS PÚBLICAS DE SONSONATE
}

\section{Resumen}

El éxito o fracaso de los cambios en educación depende en gran medida de la forma en que los diferentes participantes interpretan los cambios que se les proponen. Es por eso que este estudio caracteriza las concepciones que poseen los docentes de dos centros escolares del departamento de Sonsonate en el uso que dan a la información generada por la evaluación de los aprendizajes, dado el papel clave que juegan los docentes en el éxito o el fracaso de los estudiantes. La metodología utilizada es de tipo descriptiva fenomenológica .El estudio se realizó en dos centros educativos de Sonsonate, el C.E Lotificación San Antonio del Municipio de Sonsonate y C.E Cantón El Zapote del municipio de Caluco, seleccionados el primero por ser parte del sistema integrado de Hacienda Santa Clara y haber sido apoyado por una asistencia técnica mediante implementadora externa y el segundo por tener asistencia técnica a través de los equipos técnicos locales.Los participantes fueron los docentes, directoras, estudiantes de segundo ciclo, estudiantes que abandonaron el centro educativo y los padres de familia de los estudiantes de segundo ciclo. Dentro de los principales hallazgos encontrados se ha encontrado un cambio en las tendencias a utilizar la evaluación únicamente para adjudicar calificaciones al final de un periodo didáctico y como instrumento de poder hacia los estudiantes, sino más como instrumento de apoyo a los aprendizajes.

Palabras clave: concepciones de evaluación, prácticas de evaluación, educación primaria, El Salvador. 


\title{
APPLICATION OF LEARNING ASSESSMENTS BY TEACHER IN PUBLIC SCHOOLS IN SONSONATE
}

\begin{abstract}
The success or failure of the changes in education depends substantially on how the participants involved understand the proposed changes. This study marks the conceptions teachers from the department of Sonsonate have regarding the usage of the information generated by assessments, as well as the results from teacher's contribution to the success or failure of students.

The methodology used is descriptive phenomenological. The study was conducted in two public schools in Sonsonate, C.E Lotificación San Antonio in the Municipality of Sonsonate and C.E Cantón El Zapote in the municipality of Caluco. Both schools were selected because of the following features: the first one, because it is part of the integrated system of Hacienda Santa Clara and it receives technical support through external provider. The second school was selected because it receives technical support from the local technical crew. The participants of this study were teachers, principals, students of second cycle, students who dropped out school, and parents of students in second cycle. Among the main findings, we have the change in the tendency to use the evaluation just to get a score at the end of a didactic term as well as a power tool over students, but as a learning support tool.
\end{abstract}

Key Words> Conceptions of assessments, practice of evaluations, primary school, El Salvador

\section{Introducción}

Este artículo se enmarca dentro de una investigación que se realizó en dos Centros Educativos del departamento de Sonsonate sobre las concepciones que poseen los docentes de dos Centros Escolares sobre sus prácticas de evaluación de los aprendizajes.

En este estudio se exploró el concepto de evaluación de los aprendizajes que poseen los docentes, el momento en que tiene lugar la evaluación, grado de participación que tienen los estudiantes en la definición de criterios a evaluar, procedimientos utilizados para evaluar, criterios que se usan para evaluar, justificación de los criterios utilizados, usos que se da a la información generada por la evaluación, lo que se toma en cuenta a la hora de calificar y el significado de poner una calificación. En este artículo se ha seleccionado el aspecto "Usos que dan los docentes a la información generada por la evaluación de los aprendizajes". Todo esto se realiza con la intención de comprender las concepciones que poseen los 
docentes en esta área del conocimiento pedagógico.

El estudio que se informa en esta ocasión propone aproximarse a esta práctica en su contexto natural, tal y como sucede en dos centros escolares del departamento de Sonsonate para interpretar el sentido y el uso que los docentes hacen de los datos que obtienen de la evaluación de los aprendizajes.

La evaluación de los aprendizajes ha tenido a lo largo de la historia educativa cambios importantes. Sin hacer una extensa cronología sobre el tema hay que recordar que a principios de la década de los noventa la evaluación se enfocaba en objetivos, conocimientos, experiencias, progreso, dificultades, limitaciones, tipos y grados, todo esto según los Lineamientos para la evaluación de los aprendizajes de parvularia, básica y media (1999).

En 2007 el Ministerio de Educación de El Salvador, puso a disposición de los docentes el documento Evaluación al servicio de los aprendizajes, con el que se pretendía cambiar el enfoque de evaluación por objetivos a evaluación por

\section{Antecedentes}

Han sido muchos los aportes con la intención de develar los acontecimientos cotidianos de las aulas. El estudio sobre las concepciones de los docenteshistóricamente se hizo a partir del paradigma proceso producto que concibe las aulas como una caja negra, este paradigma abraza la idea que el éxito de los aprendizajes consiste en garantizar la buena aplicación de estrategias y técnicas que el docente competencias desde una perspectiva constructivista.

No obstante, los esfuerzos y orientaciones sobre este tema, existe interés por comprender la forma en que los docentes resuelven las situaciones problemáticas relativas al proceso evaluativo y qué uso se le da a la información que generan las evaluaciones.

Desde una perspectiva economicista en un estudio realizado por el departamento de presupuesto escolar del Ministerio de Educación (Cerna, 2011) entre los años 2009, 2010 y 2011 reprobaron globalmente desde básica hasta educación media 67,$787 ; 73,013 ; 72,485$ estudiantes respectivamente a nivel nacional, lo que representa costos que oscilan entre los cuatro y un poco más de los cinco millones de dólares por año. Cerna menciona en este informe que la evaluación de los aprendizajes aunque está orientada por documentos ministeriales reconoce que en realidad está impregnada por subjetivismo del docente que evalúa.

debe conocer y dominar para obtener buenos resultados, estas técnicas y estrategias metodológicas son pensadas externamente al centro escolar $y$ concebidas para que el docente las aplique únicamente.

Estos modelos proceso-producto de acuerdo con Gimeno (1988), han demostrado su inaplicabilidad, dado que la experiencia demuestra que cuando el docente se enfrenta a problemas 
empíricos echa mano de sus propios recursos cognitivos y resuelve los problemas en concordancia con ello. Muchas son las pruebas en que se ha observado que a través de agentes externos, llámense delegados educativos, supervisores, asesores pedagógicos, asistentes pedagógicos, se han hecho recomendaciones 0 exigencias a los docentes y seestablecen compromisos, se constata que se siguen realizando las mismas prácticas que se comprometió cambiar.

Posteriormente a las teorías procesoproducto, de acuerdo con Dahlof y Lundgren citados por Barquin J (1991) sobre como los docentes utilizaban un grupo de referencia para determinar si continuaban con otra lección o si reforzaban la misma, que en su momento se llamó el Steering Group, que es básicamente un grupo directivo $o$ de referencia para determinar los avances del grupo. Ruiz menciona que con este antecedente se dio inicio a una nueva corriente que comenzó a estudiar las concepciones que poseían los docentes sobre su trabajo en las escuelas y las aulas.

En principio estos estudios del aula comprendieron, de acuerdo con Cruz, (1998) las actividades previas a la planificación didáctica de la clase, el desarrollo de la clase propiamente dicha, que comprende las interacciones que se dan en la clase, y las concepciones sobre la evaluación de los aprendizajes.

Estos aportes son valiosos desde el enfoque cognitivo, sin embargo una de las principales críticas que este paradigma recibe, es el hecho de estar sustentado teóricamente en la racionalidad técnica, la cual concibe la idea de que cualquier profesional que se
Estos escenarios comenzaron a ser estudiados desde una perspectiva cognitivista que es una concepción científica que se caracteriza de acuerdo con Gimeno (1988) por ser un modelo centrado en el profesor cuya principal pretensión es descubrir las variables cognitivas que condicionan su actuación docente y que en consecuencia influyen en los resultados de la enseñanza, esta corriente se reduce fundamentalmente al análisis de los procesos formales de índole cognitiva y psicologista. Este paradigma siguiendo a Gimeno ha tenido su máxima representación en el Institute for research of teaching de la Universidad de Michigan, se dice que los autores más destacados de esta corriente se encuentra Clark, Yinger, Paterson, Shavelson, Shulman, Vishonhaler y Cohen. Publicándose sus primeros trabajos en 1975 y aparecen ininterrumpidamente hasta nuestros días.

Dentro de esta corriente cognitivista encontramos hallazgos importantes como el descrito por Machiarola (2002) que cita a Pozo y Rodrigo lo que ellos llamaron variabilidad cognitiva inter sujeto e intra sujeto que sostienen que los formatos representacionales varían según el tipo de demanda y según el contexto de su uso, significa que las personas dan respuestas diferentes ante una misma tareapresentada de diferentes formas 0 en diferentes momentos o con metas diferentes.

enfrente a resolver problemas prácticos se caracteriza por la utilización de técnicas, destrezas, y procedimientos derivados de las proposiciones del conocimiento científico aplicable. Esto significa en relación con los problemas relativos a la evaluación de los 
aprendizajes, solo es cuestión de que el docente aplique de manera adecuada las soluciones producidas por los descubrimientos científicos. Gimeno (1988) y Barquín (1991) coinciden en lo vano de estas pretensiones, dado que los fenómenos áulicos se caracterizan por la complejidad, la incertidumbre, la inestabilidad, la singularidad y conflictos de valor, lo que obliga a pensar que en un contexto como estos el docente debe poseer características de investigador, de artista o de estratega pedagógico.

Paralelamente a estos planteamientos cognitivistas, Barquín (et al) surge un nuevo enfoque conocido como enfoque alternativos, los cuales se dividen en dos perspectivas: El primero de ellos el enfoque fenomenológico-cualitativo, el cual considera que el comportamiento de alumnos y profesores es más de lo que puede ser deducido de los hechos externos y comportamientos observables y debe interpretarse desde la percepción $y$ los significados de los agentes. No importa tanto los hechos en si como objeto de conocimiento, sino como expresión de significados de quienes los vivencia. No basta con observar los hechos que ocurren en el aula o en la escuela, es necesario penetrar hasta la estructura de significados que los docentes dan a sus actuaciones para transformarlas.

Otra corriente que intenta comprender las actuaciones de los docentes es la conocida como perspectiva ecológiconaturista que entiende que el

Explicar las concepciones que guían a los docentes en la toma de decisiones y para interés concreto de este estudio en sus prácticas de evaluación de los aprendizajes.

REVISTA CIENCIA, CULTURA Y SOCIEDAD Vol. 5, n. 1, enero-junio/ 2018. ISSN 2305-7688 comportamiento humano se encuentra condicionado de forma compleja por el medio donde se produce. El ambiente físico y sicosocial del aula puede considerarse un ecosistema de relaciones, e interacciones de intercambios simbólicos que determinan de forma compleja el pensamiento, y la conducta del individuo y del grupo, menciona Barquín, y por lo tanto los procesos de enseñanza aprendizaje deben estudiarse en el medio natural, en el contexto complejo, ambiguo y cambiante del aula.

La evaluación tendrá que considerar tanto el resultado de las interacciones entre los participantes como los intercambios de estos con el medio, eso significa de acuerdo con este mismo autor que en cada situación personal concreta de un docente pueden predominar unos $u$ otros componentes, teorías 0 creencias, mitos 0 generalizaciones empíricas, ideas explicitas 0 resabios ideológicos inconscientes.

Se coincide con Barquín que el conocimiento y la comprensión de las peculiaridades específicas del propio conocimiento práctico es la condición del desarrollo autónomo profesional del profesor, la base del control racional de la propia actuación.

Estos marcos teóricos proceso-producto, cognitivo, fenomenológico-cualitativo, ecológico naturista ha servido de referencia para efectuar variadas investigaciones que intentan

Es importante destacar que el concepto de evaluación que se toma como referente teórico en el estudio, está relacionado con lo planteado en Evaluación al servicio de los aprendizajes y el desarrollo, MINED 
(2014), Modelo de Escuela Inclusiva de Tiempo Pleno en El Salvador, MINED (2016) en el cual se concibe una evaluación continua y sistemática a lo largo de las acciones del proceso de enseñanza y aprendizaje que permita identificar el grado de desarrollo y competencia alcanzado en todas las áreas de la formación integral del estudiante. Además dichos documentos sugieren que en ese sentido la atención del docente deja de estar privilegiando lo que el enseña por tanto deja de ser un modo de constatar lo que el estudiante ha captado de la enseñanza para pasar a ser una herramienta que permita comprender y aportar a un proceso integral.

Es por ello que esta investigación se aproxima a la subjetividad del docente con objeto conocer y comprender sus concepciones en relación al uso que los docentes dan a la información generada por la evaluación de los aprendizajes

\section{Metodología}

Respecto a la naturaleza de la investigación es de tipo descriptiva fenomenológica, se orienta en las experiencias individuales subjetivas de los participantes, Merlino A. (2009), busca conocer el significado, la estructura ideológica de la experiencia vivida por un grupo de personas en relación con un fenómeno determinado.

\section{Diseño de investigación}

El diseño del estudio es cuantitativo en el análisis de los aportes de los estudiantes y cualitativo para analizar las entrevistas con docentes. En la fase cuantitativa se hizo un sondeo basado en una encuesta a estudiantes desegundo ciclo de los dos Centros educativos, de un total de 194 se encuestaron 141 estudiantes a través de un cuestionario sobre experiencias vividas en evaluación de los aprendizajes la cual se contrastó con la teoría preexistente. Se elaboró una valoración y reflexión sobre los resultados de la encuesta. La encuesta se validó con antelación con estudiantes de segundo ciclo en un Centro educativo de similares condiciones a los centros en que se aplicó el estudio, para el caso el Centro Educativo El Cega del Municipio de Izalco.

En el aspecto cualitativo se basa en el modelo de Nevo, que utilizó Vergara C. (2011) enriquecido con otras categorías que parten de la experiencia del investigador. Se emplearon técnicas de investigación cualitativa como la observación, las entrevistas en profundidad y los grupos focales.

Se hicieron dos grupos focales con nueve estudiantes que han abandonado el sistema educativo en cada Centro Educativo. Dos grupos focales con los padres de familia de estudiantes de segundo ciclo, entre ocho y diez padres de familia en cada centro educativo independientemente que los hijos hallan reprobado o no, pues únicamente interesó conocer las experiencias vividas que tuvieron en relación a la evaluación de los aprendizajes.

Con respecto a los Directores, se realizaron dos entrevistas en profundidad, se buscó identificar las experiencias vividas y practicadas de evaluación de los aprendizajes. Las 
guías de entrevistas dirigidas a docentes y directores se sometieron al juicio de expertos, con asistentes pedagógicos con experiencia en docencia universitaria

\section{Análisis de resultados}

En cuanto al uso que los docentes dan a la información que genera la evaluación de los aprendizajes se encontraron dos sub categorías siendo estas: Primer aspecto encontrado, los resultados de la evaluación sirven para saber y reflexionar en lo que el alumno está aprendiendo y adjudicar una calificación.

En un centro se encontró que dos docentes mencionaron que la información que surge de la evaluación se usa para saber cuánto ha aprendido el niño o cuanto se ha dado a entender el maestro y, además, señala que le sirve para autoevaluar su práctica.

Otro docente menciona que le sirve para reflexionar sobre el rendimiento académico de sus estudiantes y reflexionar críticamente sobre sus prácticas, conocer las causas por las cuales los estudiantes a veces salen mal, un docente lo dice así, "Bueno en primer lugar, nos sirve para reflexionar sobre el rendimiento académico de los estudiantes, conocer cuáles son las causas para la cual a veces salen mal, en este caso hay varios jóvenes que nos preocupa que no salen mal solo enuna disciplina, sino que reprobaron la mayoría de disciplinas y también otro aspecto importante acá es que sirve para reflexionar directamente sobre nuestras prácticas, o sea, ¿qué estamos y asistencia pedagógica a Centros Escolares.El estudio fue dirigido por el investigador principal y el apoyo de un estudiante de sociología. nosotros haciendo en el aula, si lo estamos haciendo bien o mal, o sea, de qué manera nosotros estamos fallando para poder mejorar?". Una maestra señaló que la evaluación sirve para tener conocimientos más específicos de los aprendizajes de los estudiantes.

La tabla No. 1 muestra comparativamente los usos de la información generada por la evaluacion: 


\begin{tabular}{|c|c|c|c|}
\hline C.E. & Categorías & $\begin{array}{l}\text { Saber, reflexionar, conocer los } \\
\text { aprendizajes de los estudiantes. }\end{array}$ & $\begin{array}{l}\text { Poner una nota o alcanzar los } \\
\text { objetivos previamente definidos }\end{array}$ \\
\hline \multirow{3}{*}{$\begin{array}{l}\text { C. } \\
\text { E.Lot. } \\
\text { San } \\
\text { Antonio }\end{array}$} & Docente 1 & $\begin{array}{l}\text {."Me arroja información para saber } \\
\text { cuánto ha aprendido el niño, cuanto me } \\
\text { he dado a entender, y aun para } \\
\text { autoevaluarme en que he fallado" }\end{array}$ & \\
\hline & Docente 2 & $\begin{array}{l}\text {.."Nos sirve para reflexionar sobre el } \\
\text { rendimiento académico de los } \\
\text { estudiantes, conocer cuáles son las } \\
\text { causas para la cual a veces salen mal, } \\
\text { es para reflexionar directamente con } \\
\text { nuestras prácticas.:."” }\end{array}$ & \\
\hline & Docente 3 & & $\begin{array}{l}\text {... "me sirve para poner una nota, } \\
\text { para conocer los niños que han } \\
\text { logrado las competencias..." }\end{array}$ \\
\hline \multirow[t]{3}{*}{$\begin{array}{l}\text { C. E. } \\
\text { Cantón } \\
\text { El } \\
\text { Zapote }\end{array}$} & Docente 4 & $\begin{array}{l}\text { “..Conocimientos más específicos del } \\
\text { nivel de aprendizaje de los } \\
\text { estudiantes... Nos sirve para } \\
\text { evaluarnos a nosotros mismos en que } \\
\text { estamos fallando y que estamos bien, } \\
\text { pero también nos sirve para hacer un } \\
\text { plan de refuerzo..." }\end{array}$ & \\
\hline & Docente 5 & & $\begin{array}{l}\text {..."sirve para ver si en realidad } \\
\text { estamos alcanzando los objetivos que } \\
\text { se proponen en la materia el docente } \\
\text { o el MINED..." }\end{array}$ \\
\hline & Docente 6 & & $\begin{array}{l}\text {..."Esa información va en las } \\
\text { actividades diarias, las actividades } \\
\text { cotidianas, ahí va empleado, porque } \\
\text { eso al final del trimestre ahí esta y } \\
\text { eso a los niños les ayuda bastante..." }\end{array}$ \\
\hline
\end{tabular}

Tres docentes entrevistados expresaron que utilizan la evaluación para investigar los estilos, ritmos y formas de aprender de los estudiantes, aunque no los mencionaron explícitamente, ni mostraron la caracterizaron de estos estilos de aprendizaje en sus estudiantes, si mostraron ejemplos de las adaptaciones curriculares que realizan producto de las observaciones a los estudiantes.

En un segundo aspecto encontrado en otros tres docentes entrevistados es que la información generada por la evaluación sirve para colocar una nota y conocer lo que los estudiantes han se expresan estas mejoras en la planificación escolar. logrado de la competencia, una maestra lo menciona de la siguiente manera: Bueno en primer lugar eso me sirve para poner una nota, para conocer los niños que han logrado las competencias".

Por su parte las directoras de los centros educativos manifiestan que la información que se extrae de las evaluaciones en general se utiliza para identificar las formas de aprender de los estudiantes pero referidos estos aprendizajes a cuestiones procedimentales o conceptuales que los estudiantes deben lograr, además expresan que les sirve para mejorar su práctica, pero no se mostró en qué áreas 
Se consideró importante conocer las opiniones de estudiantes que por diversas razones abandonaron la escuela con el propósito de descubrir si existe una tendencia intergeneracional en los usos que se hace de la información que genera la evaluacion de los aprendizajes. Las opiniones de estos estudiantes y la opinión de los padres de familia, difieren de lo manifestado por los docentes y directoras.

Debe tomarse en cuenta que estos grupos pertenecen a otras generaciones cuyas experiencias en evaluación difiere de lo que ocurre actualmente en la escuela.

La tabla No. 2 muestra comparativamente lo expresada por los estudiantes que han abandonado y de padres de familia.

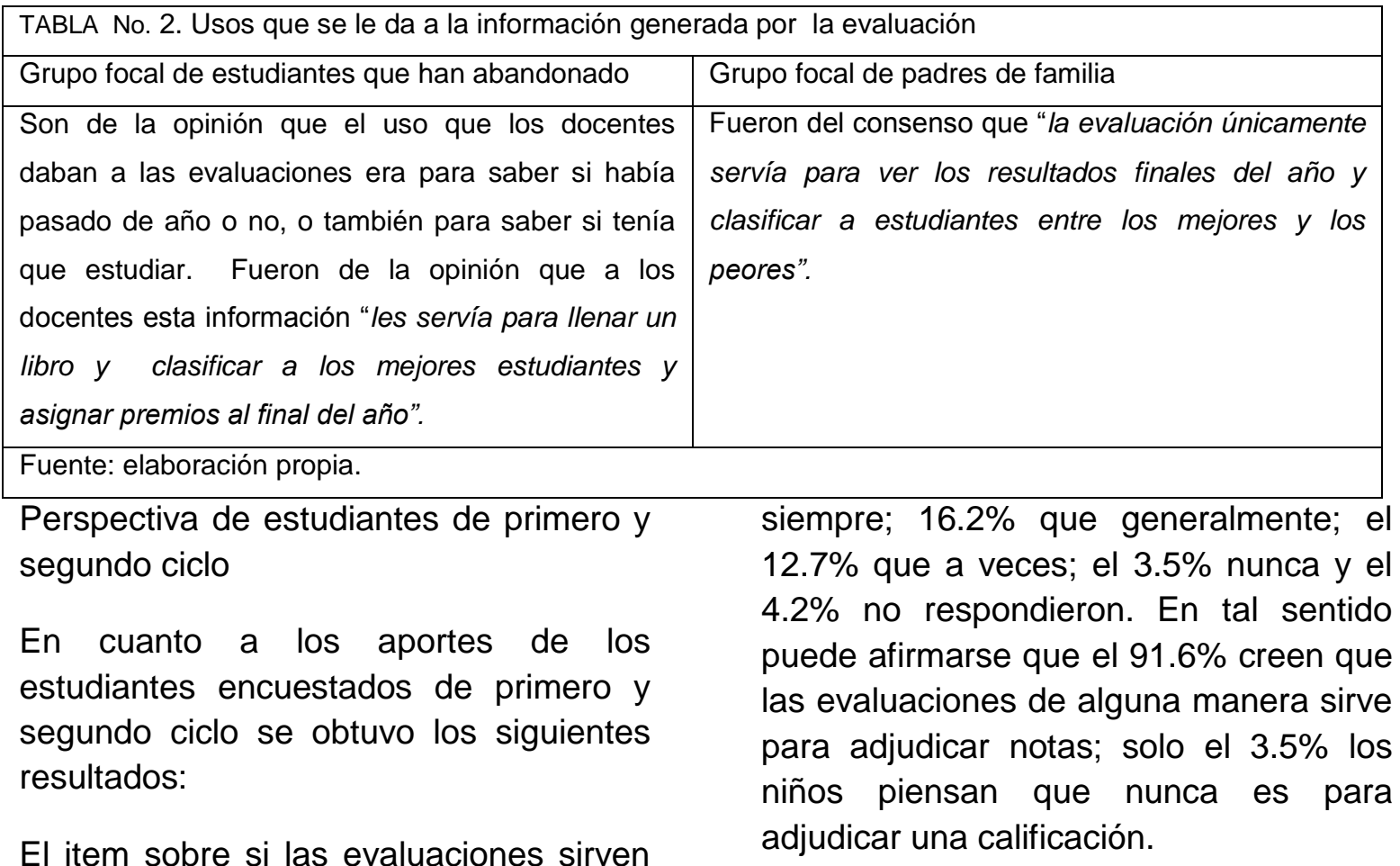

únicamente para adjudicar una

calificación, el $62.7 \%$ respondieron que

Las evaluaciones sirven normalmalmente para adjudicar una calificacion

\begin{tabular}{|ll|r|r|r|r|}
\hline & & & $\begin{array}{c}\text { Porcentaje } \\
\text { válido }\end{array}$ & $\begin{array}{r}\text { Porcentaje } \\
\text { acumulado }\end{array}$ \\
\hline Válidos & siempre & 89 & 62.7 & 63.1 & 63.1 \\
& generalmente & 23 & 16.2 & 16.3 & 79.4 \\
& a veces & 18 & 12.7 & 12.8 & 92.2 \\
& nunca & 5 & 3.5 & 3.5 & 95.7 \\
& no responde & 6 & 4.2 & 4.3 & 100.0 \\
Perdidos & Total & 141 & 99.3 & 100.0 & \\
Total & Sistema & 1 & .7 & & \\
\hline
\end{tabular}

Fuente: Elaboración propia 
En la pregunta sobre si la evaluación servía para castigar la falta de dedicación de los estudiantes, el $26.1 \%$ respondieron que siempre; $5.6 \%$ generalmente: $30.3 \%$ a veces; $35.2 \%$ nunca y el $2.1 \%$ no responde.
Un buen porcentaje de estudiantes $65.5 \%$ perciben que las evaluaciones no se utilizan para castigar, el $26.1 \%$ perciben la evaluacion como instrumento de castigo

En ocaciones las evaluaciones han servido 'para castigar mi falta de dedicacion

\begin{tabular}{|ll|r|r|r|r|}
\hline & & & $\begin{array}{c}\text { Porcentaje } \\
\text { válido }\end{array}$ & $\begin{array}{r}\text { Porcentaje } \\
\text { acumulado }\end{array}$ \\
\hline Válidos & siempre & 37 & 26.1 & 26.2 & 26.2 \\
& generalmente & 8 & 5.6 & 5.7 & 31.9 \\
& a veces & 43 & 30.3 & 30.5 & 62.4 \\
& nunca & 50 & 35.2 & 35.5 & 97.9 \\
& no responde & 3 & 2.1 & 2.1 & 100.0 \\
& Total & 141 & 99.3 & 100.0 & \\
Perdidos & Sistema & 1 & .7 & & \\
Total & & 142 & 100.0 & & \\
\hline
\end{tabular}

Fuente: Elaboración propia

Se preguntó si el profesor apoya a los estudiantes de acuerdo con el resultado obtenido en los exámenes, el $52.8 \%$ opina que siempre; el $6.3 \%$ generalmente; $26.8 \%$ a veces; $12.7 \%$ nunca y $0.7 \%$ no responde. Los niños tienen la idea que los docentes les apoyan de acuerdo con los resultados obtenidos en las evaluaciones esta misma opinión es compartida por los padres de familia entrevistados en los grupos focales, quienes opinan que efectivamente se nota que los docentes se preocupan por los alumnos, -"yo me he fijado que los maestros hoy si se preocupan por los alumnos"- Este es un comentario que hicieron padres de familia tanto del Centros Escolar El Zapote como del Centro Escolar Lotificación San Antonio.

Normalmente mi profesor me apoya de acuerdo a como son mis resultados en los examenes

\begin{tabular}{|ll|r|r|r|r|}
\hline & & & $\begin{array}{c}\text { Porcentaje } \\
\text { válido }\end{array}$ & $\begin{array}{r}\text { Porcentaje } \\
\text { acumulado }\end{array}$ \\
\hline Válidos & siempre & Frecuencia & Porcentaje & 53.2 \\
& generalmente & 75 & 52.8 & 53.2 & 59.6 \\
& a veces & 9 & 6.3 & 6.4 & 86.5 \\
& nunca & 38 & 26.8 & 27.0 & 99.3 \\
& no responde & 18 & 12.7 & 12.8 & 100.0 \\
& Total & 1 & .7 & .7 & \\
Perdidos & Sistema & 141 & 99.3 & 100.0 & \\
Total & 1 & .7 & & \\
\hline
\end{tabular}

Fuente: elaboración propia 


\section{Discusión de resultados}

Al comparar estos resultados con otros estudios como el realizado con docentes destacados de educación básica en la Comuna Curicó república de Chile, Vergara C.R. (2011) menciona más similitudes que diferencias, especialmente en lo que respecta a su conceptualización, funciones y finalidades.

Los docentes sostienen que la evaluación es una tarea motivadora y pedagógica, expresan que es a través de la evaluación que les es posible mejorar sus metodologías de enseñanza, enfatizan su aspecto formativo y la necesidad de abrirse a una mayor participación de los estudiantes, reconocen la importancia que tiene la participación del alumno en la evaluación de los aprendizajes, esto se contrapone a ciertas teorías que afirman que los docentes consideran que efectuar planeamiento de evaluación es una carga más que les impide atender de mejor manera a los estudiantes

Otro aspecto relevante encontrado en la investigación de Vergara es que, usan la evaluación como una herramienta de control disciplinario, lo que deja en evidencia que la evaluación es concebida como medición, y que se equipara con calificaciones de carácter cuantitativo.

Otros estudios como el de Contreras (2008) que hace una rigurosa compilación de estudios internacionales en los que cita a Celman (2005); Litwin (2005); Camiloni (2005); Darling-Hamond (2001); Eisner (1994) sobre las concepciones de los docentes en evaluación de los aprendizajes, menciona entre sus conclusiones que la evaluación se sigue considerando desvinculada de los aprendizajes y destinada únicamente a medir, a acreditar y a certificar.

Como se ha visto en esta investigación en escuelas públicas de Sonsonate, han participado estudiantes que actualmente son atendidos en la escuela, estudiantes que han abandonado las aulas, docentes y padres de familia, en los cuales se ha podido contrastar y tomando en cuenta la diferencia generacional entre padres de familia $y$ estudiantes que han abandonado que los usos que se le da a los resultados de la evaluacion es la misma, es decir, que ha servido únicamente para medir, segregar a los buenos de los malos estudiantes y que además ha servido para premiar a los mejores estudiantes al finalizar el año lectivo.

Sin embargo es de notar y de acuerdo con los datos mostrados, hay aquí una tendencia de cambio sobre estas concepciones instrumentalistas en esta generación, tanto estudiantes como padres de familia reconocen los esfuerzos de los docentes en el nuevo uso que se la da a los resultados de las evaluaciones. Las variadas formas que se usan para evaluar permiten al docente conocer mejor a sus estudiantes y con ello la realización incipiente de adaptaciones curriculares permite de mejor manera acompañar los aprendizajes de los estudiantes. 


\section{Conclusión}

Lo anterior permite afirmar que dado que los docentes de los centros educativos participantes desarrollan diferentes tipos de metodologías activas y que han participado de las diferentes formas de apoyo pedagógico, están evaluando con un enfoque constructivista los aprendizajes de sus estudiantes. Se concluye que se ha trascendido la tradicional prueba objetiva como único instrumento de evaluación por una variedad de instrumentos y técnicas de evaluación que hoy se aplican.

La triangulación de la información encontrada permite afirmar que, aunque los docentes aun no utilizan la información generada por las evaluaciones para caracterizar a sus estudiantes de forma sistemática, están apoyando a sus estudiantes de diferentes formas en sus estilos ritmos y formas de aprender, lo cuales percibido por los estudiantes de segundo ciclo y por los padres de familia.

Este aspecto difiere de lo encontrado por la asistencia técnica en 2010.En este informe se expresa que la evaluación es considerada por los docentes, como un acto al final de un periodo determinado y no como un proceso formativo de apoyo a los diferentes estilos, formas y ritmos de aprendizaje de los estudiantes.

Del mismo modo difiere de lo percibido por los estudiantes que han abandonado $y$ por los padres de familia $y$ es coherente en buena medida con la teoría expresada en Orientación al servicio delAprendizaje y el desarrollo MINED (2014).En la cual se menciona que "la evaluación es un proceso de recopilación, organización e interpretación de información obtenida mediante diferentes técnicas e instrumentos con la finalidad de valorar y emitir juicios de valor sobre los aprendizajes de los estudiantes y tomar decisiones que mejoren el proceso de aprendizaje".

Significa que la evaluación debería servir para conocer cómo aprende los estudiantes, para que el docente diseñe en coherencia las estrategias metodológicas que se adecuen al estilo y forma de aprender y en ningún momento debería generar nerviosismos o ser utilizada como mecanismo de poder o cohesión hacia los estudiantes.

\title{
FUENTES DE INFORMACION CONSULTADAS Fuentes primarias
}

\begin{abstract}
Libros de promoción de estudiantes de C.E Lotificación San Antonio y C.E C/ El Zapote, año 2014. Elaborado por los docentes del centro educativo.

Libros de registro de evaluaciones de docentes de C.E Lotificación San Antonio y C.E C/ El Zapote, año 2014. Elaborado por docentes del centro educativo

Listas de cotejo, listas de observación, organizadores gráficos, cuadernos de estudiantes, material producido por estudiantes. Técnicas de evaluacion de los aprendizajes utilizadas por los docentes del centro educativoC.E Lotificación San Antonio y C.E C/ El Zapote.

Vega, J. E. (2010). Informe de trabajo de asistente tecnico pedagogico Direccion Departamental de Educacion de Sonsonate.Informe tecnico.
\end{abstract}


Saúl Ángel Ventura. Asistente pedagógico de la Dirección Departamental de Educación de Sonsonate.

Maestro en metodología de la investigación científica Universidad Evangélica de El Salvador. P. 6-18

\section{Fuentes secundarias}

Alicia Inciarte. Generación de Teoría Fundamentada.

Universidad de Zulia. Facultad de Humanidades y Educación.(2011)

http://www.eduneg.net/generaciondeteoria/files/INFORMETEORIA FUNDAMENTADA.pdf.

Carazo, P. C. pensamiento y gestion. Revista de la division de ciencias administrativas de la Universidad del Norte, 165193.(2006)

http://dialnet.unirioja.es/servlet/articulo?codigo $=2499471$

Cerna, H. E. Serie historica de la repeticion (2011). Ministerio de Educacion San Salvador.

Contreras, M. P. (2008). Concepciones que orientan las practicas evaluativas de los profesores:un problema a develar. Estudios pedagogicos de Valdivia.

Cruz, M. d. (1998). La enseñanza ejes y concepciones. Estudios pedagogicos, 31-41.

Gimeno, A. I. (4 de diciembre de 1988). Pensamiento y accion en el profesor:de los estudios sobre planificacion al pensamiento practico. Obtenido de dialnet: dialnet.uniroja.es/descarga/articulo48302.pdf

ElliothJhon(1990). Investigacion-ccion en Educación.

Ediciones Moratta:

Evaluación de los aprendizajes en la Escuela Inclusiva. Sistema Integrado de Escuela Inclusiva de Tiempo Pleno (2014).

Machiarola, V. Teorias Implicitas y formacion profesional Obtenido de Academia.edu:http://www.academia.edu/2245020/.(8 de diciembre de 2002).

Mauricio, E. El huerto como laboratorio.C.E Crio Rodesia, Sonsonate. (S. Ventura, Entrevistador)(20 de noviembre de 2013).

Merlino A. (2009) Investigacion cualitativa en ciencias sociales Buenos Aires, Cengage Learnig Argentina.

MINED. (1999). Lineamientos para la evaluacion de los aprendizajes de parvularia, basica y media. San Salvador.

MINED. (2007). Evaluacion al servicio de los aprendizajes. San Salvador.

Evaluación al servicio de los aprendizajes y el desarrollo, MINED (2014),

Modelo de Escuela Inclusiva de Tiempo Pleno en El Salvador, MINED (2016)

Ruiz, J. B. (1991). La Evolucion del pensamiento del profesor.

http://www.mecd.gob.es/dctm/revistade educacion/articulosre294/re29413.pdf?documentld=0901e72b813577da

Vergara, C. (2011). Concepciones en evaluacion de los aprendizajes de docentes destacados en educacion basica. revista electronica investigaciones educativas , 1-130.

http://redalyc.uaemex.mx/src/inicio/ArtPdfRed.jsp?iCve=44718060011

Sergio R. Bacerril Comprender la práctica docente. Editorial, plaza y Valdez, Mexico.(1999) 